\title{
Simulation study of the large-scale modification of the mid-latitude F-layer by HF radio waves with different powers
}

\author{
G. I. Mingaleva, V. S. Mingalev, and O. V. Mingalev \\ Polar Geophysical Institute of the Kola Scientific Center, Russian Academy of Sciences, Fersman Str. 14, \\ Apatity 184209, Murmansk Region, Russia \\ Correspondence to: V. S. Mingalev (mingalev@pgia.ru) \\ Received: 11 March 2011 - Revised: 23 July 2012 - Accepted: 26 July 2012 - Published: 17 August 2012
}

\begin{abstract}
A mathematical model of the ionosphere, developed earlier, is applied to investigate the large-scale midlatitude F-layer modification by HF radio waves with different powers. Simulations are performed for the point with geographic coordinates of the "Sura" heating facility (Nizhny Novgorod, Russia) for autumn conditions. The calculations are made for distinct cases, in which the effective absorbed power has different values belonging to the 5-100 MW range, both for nocturnal and daytime conditions. The frequency of powerful HF waves is chosen to be close to the most effective frequency for the large-scale F2-layer modification. The results of modeling indicate that the effective absorbed power can influence considerably the F-layer response to high-power radio waves in the mid-latitude ionosphere.
\end{abstract}

Keywords. Ionosphere (Modeling and forecasting)

\section{Introduction}

During the last four decades, experiments with high-power, high-frequency radio waves were successfully used for the investigation of the ionospheric plasma's properties. In particular, many experimental studies have been applied to the large-scale F-region modification by powerful HF radio waves and many interesting results were obtained (Utlaut and Violette, 1974; Gordon and Carlson, 1974; Mantas et al., 1981; Jones et al., 1986; Djuth et al., 1987; Duncan et al., 1988; Hansen et al., 1992; Mantas, 1994; Honary et al., 1995; Robinson et al., 1996; Frolov et al., 1997; Gustavsson et al., 2001; Rietveld et al., 2003; Ashrafi et al., 2007; Dhillon et al., 2007; Kosch et al., 2007; Yeoman et al., 2007; Clausen et al., 2008; Pedersen et al., 2008). It turns out that significant variations in the electron temperatures and densities can be produced by powerful HF waves in the F-layer.

Moreover, the experimental investigations were accompanied by simulation studies of the ionosphere's response to HF heating. In particular, the mathematical model of the Flayer, which can be affected by a powerful HF wave, has been developed by Mingaleva and Mingalev (1997). This model has been used to simulate the influence of the HF wave frequency and modulation regime on the expected response of the high-latitude F-layer to HF heating (Mingaleva et al., 2003, 2009). The results of simulation, obtained with the help of this model for the point with geographic coordinates of the ionospheric heater near Tromso, Scandinavia, have indicated that considerable variations in the electron temperature profiles can be produced by powerful HF waves in the highlatitude F-region. The variations of the electron temperature profiles can ultimately lead to essential decrease in the electron concentration in wide height range including the level of the F2-region peak. It was found that the strong dependence exists of heater-induced electron temperature and concentration large-scale changes at the level of the F-region peak on the incident wave frequency. To obtain the maximal effect of HF heating on the electron concentration at the level near to the F2-layer peak, the ionospheric heater has to operate at a frequency slightly less than the F2-layer critical frequency. This frequency was called the most effective frequency for the large-scale modification of the F2-layer and is denoted by $f_{\text {eff. }}$. It was established that $f_{\text {eff }}$ has distinct values for nocturnal and daytime conditions (Mingaleva et al., 2003). It should be emphasized that the introduced most effective frequency, $f_{\text {eff }}$, is connected with the very large-scale variations of the electron concentration at the level of the F2-layer. 
However, physical conditions in the high-latitude ionosphere differ from such conditions in the mid-latitude ionosphere while heating experiments are conducted. In particular, a geomagnetic field inclination at mid-latitudes is smaller than that at high latitudes. Moreover, the plasma convection of the polar ionosphere is absent at mid-latitudes. It is of interest to apply the mathematical model, developed by Mingaleva and Mingalev (1997), for investigations of the midlatitude F-layer modification by powerful HF radio waves. Such investigations have been begun in the paper of Mingaleva et al. (2008), devoted to the study of how the value of the heating frequency affects the large-scale mid-latitude F-region modification. It was established in this paper that there should exist such, most effective, heating wave frequency at which a decrease in the electron concentration at the level near to the F2-layer peak over the heating facility should be maximal. However, the results by Mingaleva et al. (2008) have been obtained for the fixed value of the effective absorbed power (EAP) which is the input parameter of the mathematical model. It is known that the EAP is connected with the effective radiated power (ERP) by the formula

$\mathrm{EAP}=\eta \cdot \mathrm{ERP}$

where $\eta$ is the coefficient characterizing the fraction of the energy of the powerful HF wave deposited in the ambient electron gas and lost for its heating. In the concrete ionospheric heating experiment, the value of the coefficient $\eta$ is not known exactly. Moreover, distinct high-power radio wave heaters can provide different values of the ERP. Therefore, in various ionospheric heating experiments, the values of the EAP may be different.

The purpose of this paper is to present the simulation results obtained with the help of the mathematical model, developed earlier, and devoted to the investigation of how the EAP affects the large-scale mid-latitude F-layer response to high-power radio waves. The calculations are made for distinct cases in which the EAP varies from 5 to $100 \mathrm{MW}$.

\section{Numerical model}

The mathematical model of the F-region ionosphere, which can be affected by a powerful HF wave, developed earlier by Mingaleva and Mingalev (1997), is utilized in the present study. The model takes into account the geomagnetic field inclination, magnetization of the plasma at F-layer altitudes, and convection of the ionospheric plasma. Charged particles of the F-region ionosphere are supposed to be strongly magnetized, and they may be considered to be attached to magnetic field lines. The ionosphere plasma drift in the direction perpendicular to the magnetic field $\boldsymbol{B}$ is strongly affected by the electric field $\boldsymbol{E}$. Therefore, the F-layer ionosphere plasma transport perpendicular to magnetic field lines follows $\boldsymbol{E} \times \boldsymbol{B}$ convection trajectories. In the model calculations, we obtain the configuration of the convection trajectory and calculate the plasma drift velocity along it at the F-layer altitude, applying a distribution of the electric field $\boldsymbol{E}$, by analogy with the study of Mingaleva and Mingalev (1996).

The model uses the electric field distribution which is the combination of the pattern $B$ of the empirical models of highlatitude electric fields of Heppner (1977) and the empirical model of ionospheric electric fields at middle latitudes, developed by Richmond (1976) and Richmond et al. (1980). In the model calculations, the temporal history is traced of the ionospheric plasma in the part of the magnetic field tube situated in the illuminated region over an ionospheric heater. The model accounts for convection moving plasma in and out of the heater beam, which is often ignored by other models and even experimental studies. A part of the magnetic field tube of the ionospheric plasma is considered at distances between $100-700 \mathrm{~km}$ from the Earth along the magnetic field line. In the present study, we assume that the part of the magnetic field tube can be in the illuminated region over an ionospheric heater during the period longer than five minutes. The parallel (to the magnetic field) plasma flow in the magnetic field tube is described by the system of transport equations, which consists of the continuity equation, equation of motion for ion gas, and heat conduction equations for ion and electron gases. These equations in the reference frame, moving together with a field tube of plasma, whose axis $h$ is directed upwards along the magnetic field line, may be written as follows:

$$
\begin{aligned}
& \frac{\partial N}{\partial t}+\frac{\partial}{\partial h}\left(N V_{\mathrm{i}}\right)=q-l \\
& m_{\mathrm{i}} N\left(\frac{\partial V_{\mathrm{i}}}{\partial t}+V_{\mathrm{i}} \frac{\partial V_{\mathrm{i}}}{\partial h}\right)-\frac{4}{3} \frac{\partial}{\partial h}\left(\mu \frac{\partial V_{\mathrm{i}}}{\partial h}\right)+\frac{\partial}{\partial h}\left[N \kappa\left(T_{\mathrm{i}}+T_{\mathrm{e}}\right)\right] \\
& \quad+m_{\mathrm{i}} N g \sin I=m_{\mathrm{i}} N \sum_{n=1}^{3} \frac{1}{\tau_{\mathrm{in}}}\left(U_{n}-V_{\mathrm{i}}\right)
\end{aligned}
$$

$$
\begin{aligned}
\frac{\partial T_{\mathrm{i}}}{\partial t} & =\frac{1}{M} \frac{\partial}{\partial h}\left(\lambda_{\mathrm{i}} \frac{\partial T_{\mathrm{i}}}{\partial h}\right)-V_{\mathrm{i}} \frac{\partial T_{\mathrm{i}}}{\partial h}+\frac{\gamma-1}{N}\left(\frac{\partial N}{\partial t}+V_{\mathrm{i}} \frac{\partial N}{\partial h}\right) T_{\mathrm{i}} \\
& +\frac{1}{M}\left(P_{\mathrm{ie}}+\sum_{n=1}^{3} P_{\mathrm{in}}\right) \\
\frac{\partial T_{\mathrm{e}}}{\partial t} & =\frac{1}{M} \frac{\partial}{\partial h}\left(\lambda_{\mathrm{e}} \frac{\partial T_{\mathrm{e}}}{\partial h}\right)-V_{\mathrm{e}} \frac{\partial T_{\mathrm{e}}}{\partial h}+\frac{\gamma-1}{N}\left(\frac{\partial N}{\partial t}+V_{\mathrm{e}} \frac{\partial N}{\partial h}\right) T_{\mathrm{e}} \\
& +\frac{1}{M}\left(P_{\mathrm{ei}}+\sum_{n=1}^{3} P_{\mathrm{en}}+Q+Q_{\mathrm{f}}-L_{\mathrm{r}}-L_{\mathrm{v}}-L_{\mathrm{e}}-L_{\mathrm{f}}\right)
\end{aligned}
$$

where $N$ is the $\mathrm{O}^{+}$ion number density (which is assumed to be equal to the electron density at the F-layer altitudes); $V_{\mathrm{i}}$ is the parallel (to the magnetic field) component of the positive ion velocity; $q$ is the photoionization rate; $l$ is the positive ion loss rate (taking into account the chemical reactions 
$\mathrm{O}^{+}+\mathrm{O}_{2} \rightarrow \mathrm{O}_{2}^{+}+\mathrm{O}, \mathrm{O}^{+}+\mathrm{N}_{2} \rightarrow \mathrm{NO}^{+}+\mathrm{N}, \mathrm{O}_{2}^{+}+e \rightarrow \mathrm{O}+\mathrm{O}$, and $\left.\mathrm{NO}^{+}+e \rightarrow \mathrm{N}+\mathrm{O}\right) ; m_{\mathrm{i}}$ is the positive ion mass; $\kappa$ is Boltzmann's constant; $T_{\mathrm{i}}$ and $T_{\mathrm{e}}$ are the ion and electron temperatures, respectively; $g$ is the acceleration due to gravity; $I$ is the magnetic field dip angle; $1 / \tau_{\text {in }}$ is the collision frequency between ion and neutral particles of type $n ; U_{n}$ is the parallel component of velocity of neutral particles of type $n$; $M=3 / 2 \kappa N, \gamma=5 / 3 ; V_{\mathrm{e}}$ is the parallel component of electron velocity (which is assumed to be equal to the parallel component of the positive ion velocity); $\mu$ is the ion viscosity coefficient; $\lambda_{\mathrm{i}}$ and $\lambda_{\mathrm{e}}$ are the ion and electron thermal conductivity coefficients; $Q$ and $Q_{\mathrm{f}}$ are the electron heating rates due to photoionization and $\mathrm{HF}$ heating, respectively; $L_{\mathrm{r}}, L_{\mathrm{v}}, L_{\mathrm{e}}$ and $L_{\mathrm{f}}$ are the electron cooling rates due to rotational excitation of molecules $\mathrm{O}_{2}$ and $\mathrm{N}_{2}$, vibrational excitation of molecules $\mathrm{O}_{2}$ and $\mathrm{N}_{2}$, electronic excitation of atoms $\mathrm{O}$, and fine structure excitation of atoms $\mathrm{O}$, respectively.

The quantities on the right-hand sides of Eqs. (4) and (5), denoted by $P_{a b}$, describe the type $a$ particle energy change rates as a result of elastic collisions with particles of type $b$, with large drift velocity differences having been taken into account. Thus, the quantities $P_{a b}$ contain the frictional heating produced by electric fields and thermospheric winds.

A three-dimensional distribution of the thermospheric wind is an input parameter of the utilized model. This distribution is obtained by analogy with the study of Mingaleva and Mingalev (1996), with the horizontal distribution of the neutral wind at $300 \mathrm{~km}$, taken from Meriwether et al. (1973), having been applied.

The model produces the time variations of the electron density, positive ion velocity, and ion and electron temperature profiles within a magnetic field tube over an ionospheric heater. These profiles of ionospheric quantities versus distance from the Earth along the geomagnetic field line are obtained by solving the system of transport equations, described above. The natural time variations of the ionospheric parameters, which can take place in the F-layer, are taken into account by the utilized numerical model.

The applied mathematical model takes into account the following heating mechanism, caused by the action of the powerful HF radio waves. The absorption of the heater wave energy is supposed to give rise to the formation of fieldaligned plasma irregularities on a wide range of spatial scales. In particular, short-scale field-aligned irregularities are excited in the electron hybrid resonance region. These irregularities are responsible for the anomalous absorption of the electromagnetic heating wave (pump) passing through the instability region and cause anomalous heating of the plasma. The rate of this anomalous heating is denoted by $Q_{\mathrm{f}}$ and included in the heat conduction equation for electron gas, Eq. (5). The concrete expression to the $Q_{\mathrm{f}}$, containing the EAP, was taken from the study by Blaunshtein et al. (1992).
This expression is of the form

$Q_{\mathrm{f}}=Q_{\mathrm{f}}^{\mathrm{m}} \exp \left\{-b^{2}\left(\frac{N-N_{\mathrm{R}}}{N_{\mathrm{R}}}\right)^{2}\right\}$

where $Q_{\mathrm{f}}^{\mathrm{m}}$ is the maximum value of the electron heat rate due to HF heating, parameter $b$ characterizes the "half-width" of the source function, and $N_{\mathrm{R}}$ is the electron hybrid resonance value of electron concentration.

From Eq. (6) it follows that the electron heat rate $Q_{\mathrm{f}}$ depends nonlinearly on the incident wave frequency $f_{0}$, and achieves the maximum value when the wave frequency $f_{0}$ is equal to the frequency of the electron hybrid resonance:

$f_{0}=\left(f_{\mathrm{N}}^{2}+f_{\mathrm{H}}^{2}\right)^{1 / 2}$,

where $f_{\mathrm{N}}$ is the plasma frequency, given by the well-known formula

$f_{\mathrm{N}}=\frac{1}{2 \pi}\left(\frac{N e^{2}}{\varepsilon_{0} m_{\mathrm{e}}}\right)^{1 / 2}$

and $f_{\mathrm{H}}$ is the electron cyclotron frequency:

$f_{\mathrm{H}}=\frac{1}{2 \pi} \frac{e B}{m_{\mathrm{e}}}$.

Here $e$ and $m_{\mathrm{e}}$ are, respectively, the charge and mass of electron, $B$ is the magnitude of magnetic field, and $\varepsilon_{0}$ is the dielectric constant of free space. The electron hybrid resonance value of electron concentration, $N_{\mathrm{R}}$, may be obtained from Eq. (7) by using the relation given by Eq. (8). It follows that

$N_{\mathrm{R}}=4 \pi^{2} \frac{\varepsilon_{0} m_{\mathrm{e}}}{e^{2}}\left(f_{0}^{2}-f_{\mathrm{H}}^{2}\right)$.

It can be seen from relation in Eq. (10) that $N_{\mathrm{R}}$ is less than the value of electron concentration corresponding to the plasma resonance, which can be expressed by the relation

$N_{0}=4 \pi^{2} \frac{\varepsilon_{0} m_{\mathrm{e}}}{e^{2}} f_{0}^{2}$.

Therefore, the level of the electron hybrid resonance is less than the level of the plasma resonance in the regular F-layer.

The maximum value of the electron heat rate due to $\mathrm{HF}$ heating, $Q_{\mathrm{f}}^{\mathrm{m}}$, is directly proportional to the EAP and inversely proportional to the electron hybrid resonance height, raised to the second power. Consequently, a necessity of knowledge of the precise value of the coefficient $\eta$, present in Eq. (1), is absent. In the utilized model, the EAP is an input parameter of the model. In the present study, calculations are made for various values of the EAP.

The expression (6), in spite of its simplicity, allows us to evaluate approximately the influence of the EAP of the incident wave on the expected large-scale mid-latitude Fregion modification. More complete details of the utilized model may be found in the studies of Mingaleva and Mingalev (1997) and Mingaleva et al. (2003, 2009). 


\section{Presentation and discussion of results}

The mathematical model of the F-region described above, which can be affected by a powerful HF wave, is applied by us for simulations of the mid-latitude F-layer modification by HF waves with different powers. Simulations are performed for the point with geographic coordinates of the "Sura" heating facility (Nizhny Novgorod, Russia), with this point having been located on both the dark and sunlit sides of the Earth. Since the obtained results are different, it is convenient to present them separately.

\subsection{Effects of heating in the dark ionosphere}

In the present subsection, we consider the temporal history of the ionospheric plasma in the magnetic field tube moving over the ionospheric heater, when it is located on the dark side of the Earth. In the initial moment, the considered magnetic field tube is assumed to be on the magnetic meridian of 01.45 MLT. It is known that the ionospheric plasma motion at F-layer altitudes has a strong tendency to co-rotate with the Earth at mid-latitudes.

It can be noted that, at high latitudes, the ionospheric plasma at F-layer altitudes convects in the direction perpendicular to the magnetic field. The speed of the plasma convection at F-layer altitudes coincides with the drift velocity of the plasma. Therefore, variations of ionospheric quantities with time are connected with their variations along the convection trajectory. The plasma convection in the polar ionosphere is mainly induced by magnetospheric electric fields, which cannot penetrate in mid-latitudes freely. The plasma convection pattern varies markedly with the interplanetary magnetic field (IMF). For southward IMF, the pattern has two vortex cells with antisunward flow over the polar cap and return flow equatorward of the auroral oval. As a consequence of the plasma convection, the duration of a powerful HF wave effect on a heated volume of plasma can not be unlimited in the high-latitude F-region ionosphere. The attainable duration depends on the heater parameters and plasma drift velocity. The volume of plasma, disturbed by a powerful HF wave, can abandon the region illuminated by an ionospheric heater before turning off the heater owing to the convection of the plasma at F-region levels.

On the contrary, the drift trajectories, around which the magnetic field tubes are carried over mid-latitudes, are close to circles, so the ionospheric plasma at F-layer altitudes drifts approximately along a fixed latitude. The drift velocity of the plasma is conditioned by the large-scale electric field whose main fraction is a corotation electric field at midlatitudes. Therefore, a ground-based ionospheric heater situated at mid-latitudes can affect a heated plasma volume for a long time.

In the present study, we suppose that the heating facility operates continuously during the period of five minutes. The calculations are performed for autumn (1 September) and high solar activity $\left(F_{10.7}=230\right)$ conditions under moderate geomagnetic activity $\left(K_{p}=3\right)$.

It is known that a natural spatial inhomogeneity of the mid-latitude ionosphere may take place, which leads to horizontal variations of ionospheric quantities even without any HF heating. Therefore, we started from obtaining the variations of calculated ionospheric quantities along the considered drift trajectory under natural conditions without a powerful HF wave effect. The results of simulation indicate that noticeable variations of calculated profiles may take place in the nocturnal ionosphere under natural conditions without HF heating. These variations will be seen in following figures.

To investigate how the value of the effective absorbed power (EAP) influences the expected ionosphere's response to HF heating, we calculate the time variations of ionospheric quantities following the turn on of the heater for different cases in which the EAP has distinct values belonging to the 5-100 MW range. The important input parameter of the numerical model is the incident wave frequency. In the present subsection, the value of the incident wave frequency is assumed to be $3.0 \mathrm{MHz}$. This value of the incident wave frequency is slightly less than the F-layer critical frequency at the initial moment and is close to the most effective frequency for the large-scale F2-layer modification considered in the study by Mingaleva et al. (2008). In this study, as was noted earlier, it was found that, to obtain the maximal effect of HF heating on the electron concentration at the level near to the F2-layer peak, the ionospheric heater has to operate at a frequency slightly less than the F2-layer critical frequency. This frequency was called the most effective frequency for the large-scale modification of the F2-layer and denoted by $f_{\text {eff. }}$ The incident wave frequency value of $3.0 \mathrm{MHz}$ is chosen according to the recommendation from the study of Mingaleva et al. (2008). Some results of the calculations for nocturnal conditions are presented in Figs. 1-4.

It can be noticed that the peak of electron concentration profile, obtained under natural conditions without heating, is close to the level of $410 \mathrm{~km}$. It appears that this level is inappropriately high. However, the fact is that the utilized neutral wind distribution, obtained using the data presented in the study of Meriwether et al. (1973), provides a meridional component of the neutral wind velocity of about $165 \mathrm{~m} \mathrm{~s}^{-1}$ at F-layer altitudes near the considered geographic point, with the direction of the velocity being equatorward. It is known that an equatorward neutral wind ought to raise the F2-layer electron concentration peak.

Simulation results, obtained for different values of the EAP, have common characteristic features. When the heater is turned on and operates, a great energy input from the powerful HF wave originates at the level where the wave frequency is close to the frequency of the electron hybrid resonance. A pronounced peak arises in the electron temperature profile at this level. The electron temperature can increase for some thousands of degrees at this peak (Fig. 1). 


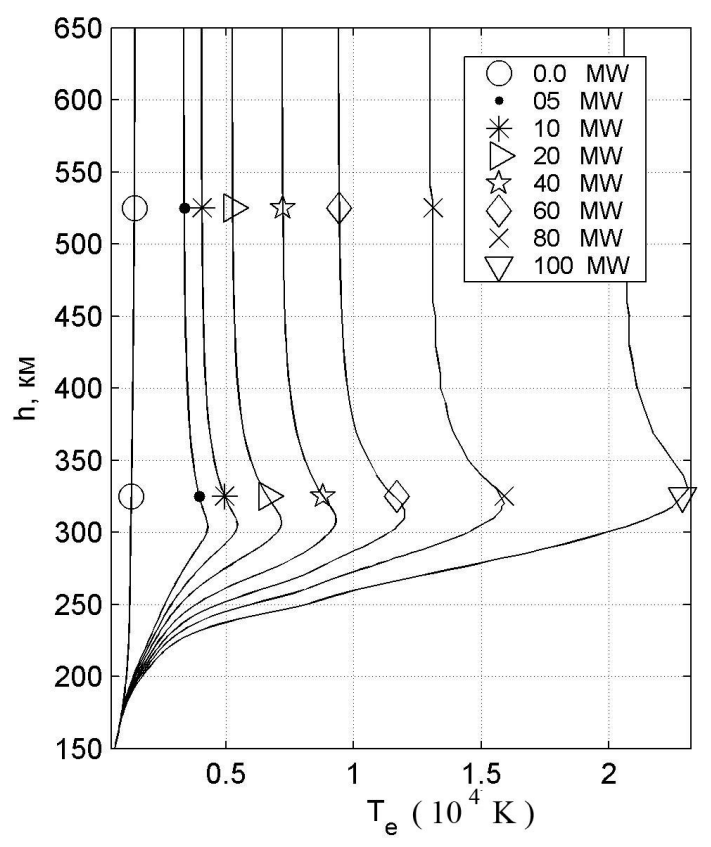

Fig. 1. Profiles of the electron temperature (in units of $10^{4} \mathrm{~K}$ ) versus distance from the Earth along the geomagnetic field line situated in the illuminated region at the moment of $300 \mathrm{~s}$ after turn on. The results are given for the incident wave frequency value of 3.0 MHz and different values of the EAP: 5, 10, 20, 40, 60, 80, and $100 \mathrm{MW}$, with symbol $0 \mathrm{MW}$ indicating the results obtained under natural conditions without heating.

This peak is situated close to the level of the electron hybrid resonance and lower than the level of the plasma resonance in accordance with Eq. (10). The increase in the electron temperature results in a rise in the electron gas pressure. From the level where the electron gas pressure peak is located, the upward and downward electron gas fluxes arise. Due to the electrical neutrality of the ionospheric plasma, the ion gas begins to move, too. Thus, ionospheric plasma fluxes arise from the level where the maximum energy input from the powerful HF wave takes place. Owing to these fluxes, a visible decrease in the electron concentration profile can arise not only near the level of maximum energy absorption from the powerful HF wave but also near the F2-layer peak (Fig. 2). After turning off the heater, the electron temperature decreases due to elastic and inelastic collisions between electrons and other particles of ionospheric plasma, and a period of recovery starts. The duration of the period of the electron temperature recovery after the HF heating is not longer than $3 \mathrm{~min}$ at F2-layer levels (Fig. 3). The duration of the period of the electron concentration recovery after the HF heating is about 10 min at F2-layer levels in the nocturnal mid-latitude ionosphere (Fig. 4). Incidentally, it is seen from results presented that the noticeable variation of the electron concentration with time (along the drift trajectory) takes place in the

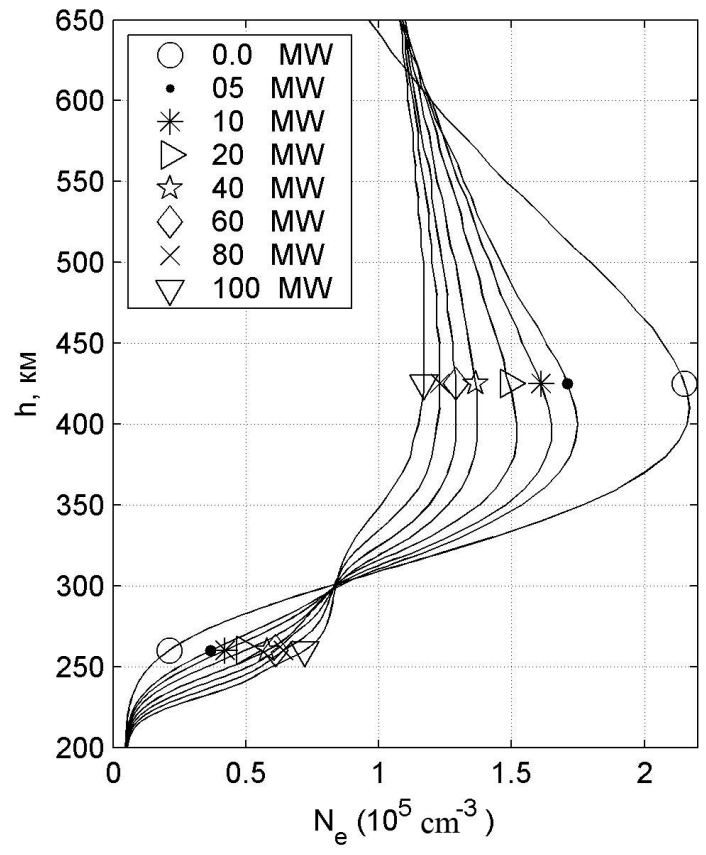

Fig. 2. Profiles of the electron concentration (in units of $10^{5} \mathrm{~cm}^{-3}$ ) versus distance from the Earth along the geomagnetic field line situated in the illuminated region at the moment of $300 \mathrm{~s}$ after turn on. The results are given for the same cases as in Fig. 1.

nocturnal mid-latitude ionosphere under natural conditions without HF heating (Fig. 4).

Simulation results indicate that appreciable variations of the electron temperature, positive ion velocity, and electron density profiles can be produced by HF heating during the period of $5 \mathrm{~min}$ in the nocturnal mid-latitude F-region, with the maximal amplitudes of variations depending significantly on the values of the EAP. It appears that the more the EAP is, the higher the values of maximal amplitudes of variations of ionospheric quantities, produced by HF heating, ought to be. Powerful HF waves should lead to a decrease of more than $47 \%$ in electron concentration at the level of the F-region peak when the EAP is $100 \mathrm{MW}$.

It can be noticed that such large depletion of the electron concentration is due to high value of the EAP included in the model calculations. It is doubtful that the EAP value of $100 \mathrm{MW}$ is attainable for the existing high-power radio wave heaters, in particular for the "Sura" heating facility (Nizhny Novgorod, Russia) whose effective radiated power (ERP) varies between 150 and $280 \mathrm{MW}$ (Belikovich et al., 1999). Nevertheless, it is not impossible that the EAP value of $100 \mathrm{MW}$ may be acquired in the future with the help of some mid-latitude ionospheric heater.

It may be recalled that the results, presented in Figs. 14 , were obtained using the incident wave frequency value of 3.0 MHz. From Fig. 2, it may be supposed that a more considerable decrease in the electron concentration profile can be obtained by utilizing a lower heating frequency. The special 


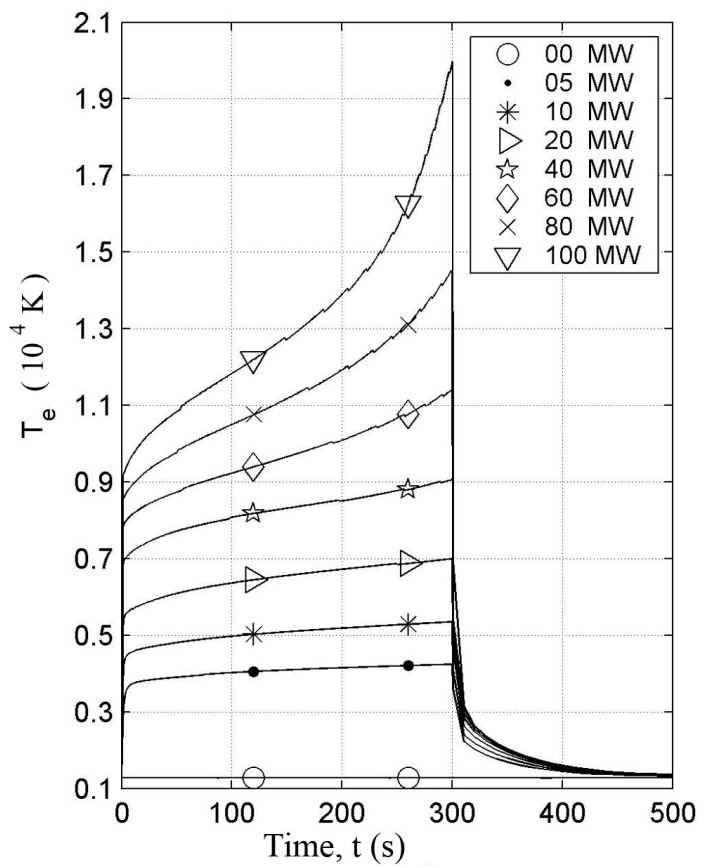

Fig. 3. The time variations of the electron temperature (in units of $10^{4} \mathrm{~K}$ ) at the level $h=300 \mathrm{~km}$. The results are given for different values of the EAP: 5, 10, 20, 40, 60, 80, and $100 \mathrm{MW}$, with symbol $0 \mathrm{MW}$ indicating the results obtained under natural conditions without heating.

study of how the value of the heating frequency affects the large-scale mid-latitude F-region modification has been carried out in the paper of Mingaleva et al. (2008). From this study, it follows that the above supposition is not correct always. The fact is that the electron hybrid resonance height depends on the heating frequency. The less the heating frequency is, the lower the electron hybrid resonance height ought to be in a regular F-layer. When the electron hybrid resonance height drops, the electron cooling rates due to elastic and inelastic collisions between electrons and neutral particles increase at this height because of an exponential rise of the neutral particles densities. If the electron cooling rates become more, the electron temperature ought to decrease. Thus, the less the incident wave frequency is, the lower the electron temperature variation amplitude, produced by the HF heating, ought to be. As a consequence, the depletion of the electron concentration may be lower. Special calculations, performed preliminary, pointed out that the incident wave frequency value of $3.0 \mathrm{MHz}$ is close to the most effective frequency for the large-scale F2-layer modification under conditions of the present subsection.

Another specific feature of the high-power HF heating process is that the heating mechanism, taken into account in the present study, is possible when the pump frequency is lower than the so-called "threshold of incident wave frequency" (TIWF), utilized in the paper by Mingaleva et al. (2003).

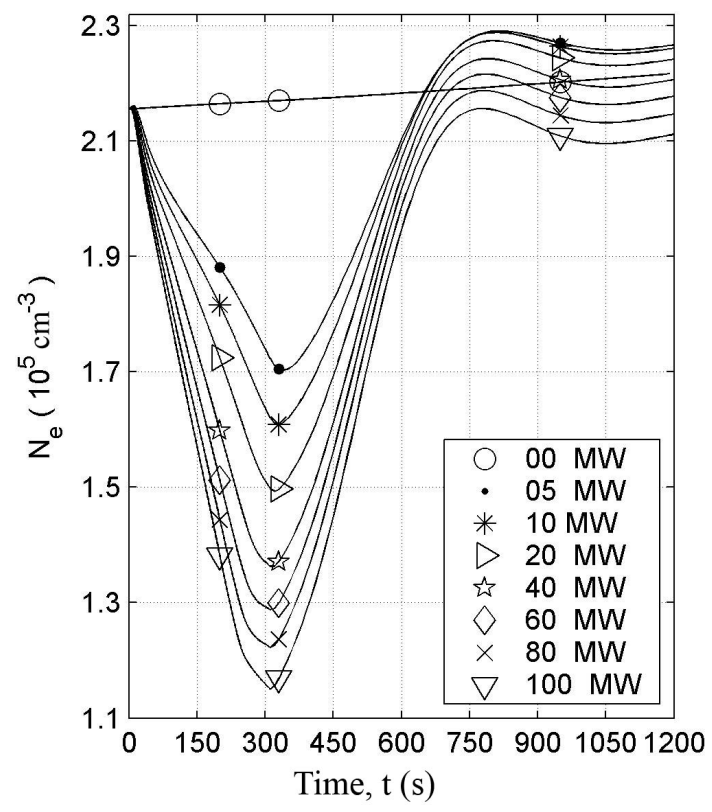

Fig. 4. The time variations of the electron concentration (in units of $10^{5} \mathrm{~cm}^{-3}$ ) at the level $h=410 \mathrm{~km}$. The results are given for the same cases as in Fig. 3.

The TIWF depends on the F-layer critical frequency and is slightly more than that. The high-power HF heating leads to a decrease in the electron concentration at the level of the Fregion peak. As a consequence, the F-layer critical frequency ought to decrease. Consequently, the TIWF is to decrease, too. Therefore, the effect of HF heating on the ionospheric plasma in the part of the magnetic field tube can be broken off due not only to the abandonment of the heater beam by it, but also to the decrease in the TIWF below the pump frequency, even though the plasma tube continues to be in the heater beam. We have avoided such events in the calculations described in the present subsection using the pump frequency value of $3.0 \mathrm{MHz}$.

\subsection{Effects of heating in the sunlit ionosphere}

Let us consider the temporal history of the ionospheric plasma in the magnetic field tube moving along the drift trajectory over the ionospheric heater, when it is located on the day side of the Earth. In the initial moment, the considered magnetic field tube is assumed to be on the magnetic meridian of 12.00 MLT. We suppose that the heating facility operates continuously during the period of five minutes. The calculations are performed for autumn (1 September) and middle solar activity $\left(F_{10.7}=130\right)$ conditions under moderate geomagnetic activity $\left(K_{p}=3\right)$.

Initially, we computed the variations of calculated ionospheric quantities along the considered drift trajectory under natural conditions without a powerful HF wave effect. These variations will be seen in following figures. It can be noticed 


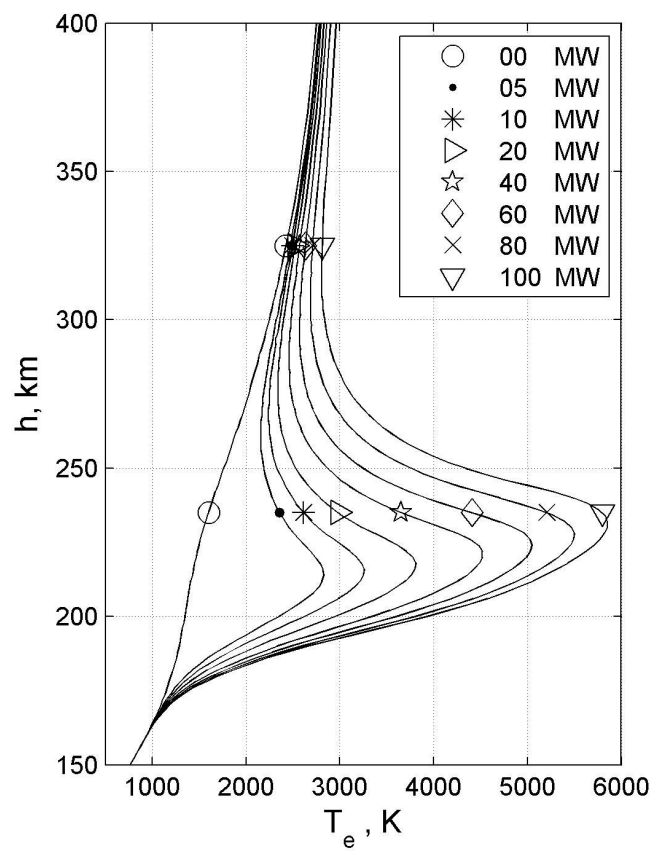

Fig. 5. Profiles of the electron temperature (in absolute degrees) versus distance from the Earth along the geomagnetic field line situated in the illuminated region at the moment of $300 \mathrm{~s}$ after turn on. The results are given for the incident wave frequency value of 7.85 MHz and different values of the EAP: 5, 10, 20, 40, 60, 80, and $100 \mathrm{MW}$, with symbol $0 \mathrm{MW}$ indicating the results obtained under natural conditions without heating.

that, on the day side, the electron concentration is more than that obtained for nocturnal ionosphere.

Next, we made a series of calculations to obtain the wave frequency which provides the maximal effect of HF heating on the electron concentration at the levels near the F2-layer peak, i.e. $f_{\text {eff }}$. It was found that $f_{\text {eff }}=7.85 \mathrm{MHz}$.

To investigate how the value of the effective absorbed power (EAP) influences the expected ionosphere's response to HF heating, we made a series of calculations, using the obtained $f_{\text {eff }}$. Some results of the calculations for daytime conditions are presented in Figs. 5-8. The results, obtained for different values of the EAP, are qualitatively very similar.

Results of simulation indicated that a great energy input from the powerful HF wave arises at the level where the wave frequency is close to the frequency of the electron hybrid resonance, when the ionospheric heater is turned on and operates. It is seen from Fig. 5 that, at this level, pronounced peaks arise in the electron temperature profiles. At these peaks, the electron temperature can increase for some thousands of degrees. These peaks lie close to the level of the electron hybrid resonance and lower than the level of the plasma resonance in accordance with Eq. (10).

As a consequence of the great increase in the electron temperature, an essential decrease in the electron concentration

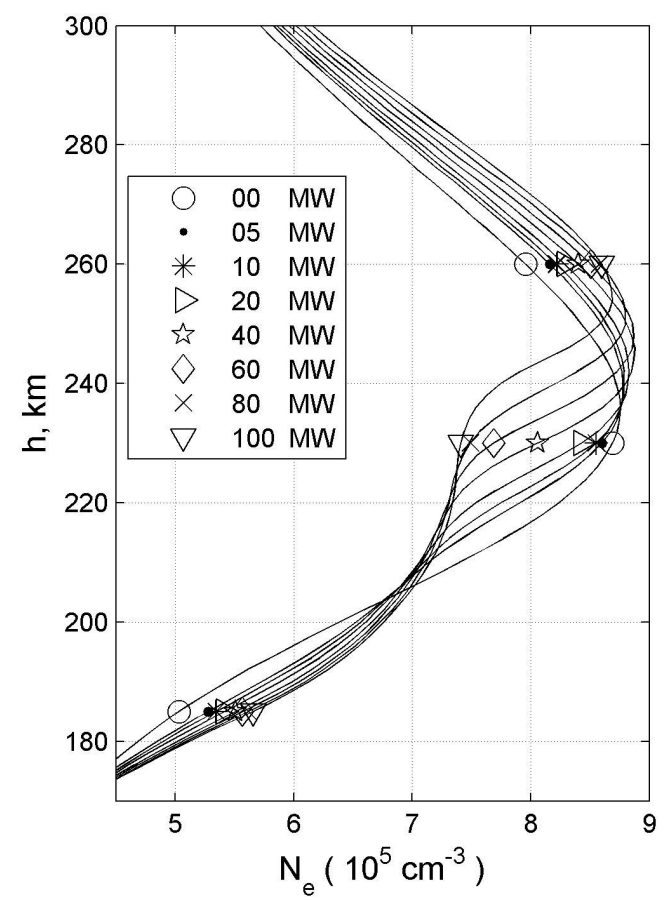

Fig. 6. Profiles of the electron concentration (in units of $10^{5} \mathrm{~cm}^{-3}$ ) versus distance from the Earth along the geomagnetic field line situated in the illuminated region at the moment of $300 \mathrm{~s}$ after turn on. The results are given for the same cases as in Fig. 5.

arises ultimately in the wide height range including the level of the F2-region peak (Fig. 6).

After turning off of the heater, the electron temperature decreases due to elastic and inelastic collisions between electrons and other particles of ionospheric plasma, and a period of recovery starts. The duration of the period of the electron temperature recovery after the HF heating is not longer than 2 min at F2-layer levels (Fig. 7). The duration of the period of the electron concentration recovery after the HF heating is about $10 \mathrm{~min}$ at F2-layer levels in the daytime mid-latitude ionosphere (Fig. 8).

The noticeable variation of the electron concentration with time (along the drift trajectory), obtained under natural conditions without a powerful HF wave effect, may be seen in Fig. 8.

It turned out that appreciable variations of the electron temperature, positive ion velocity, and electron density profiles can be produced by HF heating during the period of $5 \mathrm{~min}$ in the daytime mid-latitude F-region, with the maximal amplitudes of variations depending significantly on the values of the EAP. Simulation results indicated that the more the EAP is, the higher the values of maximal amplitudes of variations of ionospheric quantities, produced by HF heating, ought to be. Powerful HF waves should lead to a decrease of more than $13 \%$ in electron concentration at the level of the F-region peak when the EAP is $100 \mathrm{MW}$. 


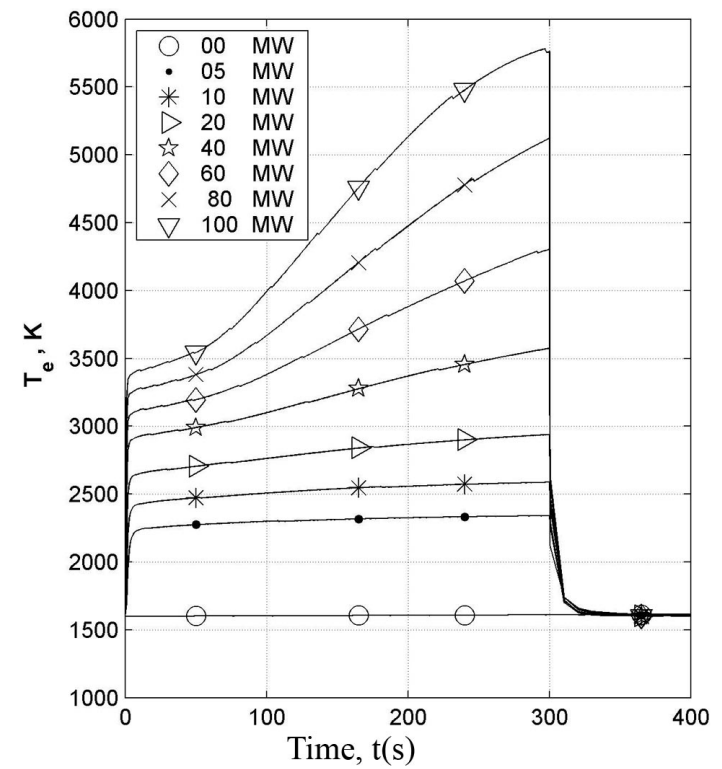

Fig. 7. The time variations of the electron temperature (in absolute degrees) at the level $h=235 \mathrm{~km}$. The results are given for different values of the EAP: $5,10,20,40,60,80$, and $100 \mathrm{MW}$, with symbol $0 \mathrm{MW}$ indicating the results obtained under natural conditions without heating.

It can be seen that the calculated changes in the large-scale structure of the daytime F2-layer are less than those obtained in the previous subsection under nocturnal conditions.

As pointed out previously, the bulk of the energy input from the powerful HF wave originates at the level where the wave frequency is close to the frequency of the electron hybrid resonance. At this level, the pronounced peak arises in the electron temperature profile. It is known that the electron hybrid resonance height is slightly less than the plasma resonance height in a regular F-layer, with the latter height being dependent on the electron concentration profile. It is evident that the electron hybrid resonance height ought to change through the variations of the electron concentration profile. As a consequence, the level, where the electron temperature profile has a peak, ought to change through the variations of the electron concentration profile. From Figs. 5 and 6, it can be seen that the simulation results produce these features in the sunlit ionosphere. Likewise, these features may be seen in the simulation results, obtained for the nocturnal ionosphere and presented in Figs. 1 and 2, even though the scales in the figures are different. Thus, despite the approximate description of the heating mechanism utilized in the present study, the simulation results reproduce correctly some essential effects of high-power HF heating.

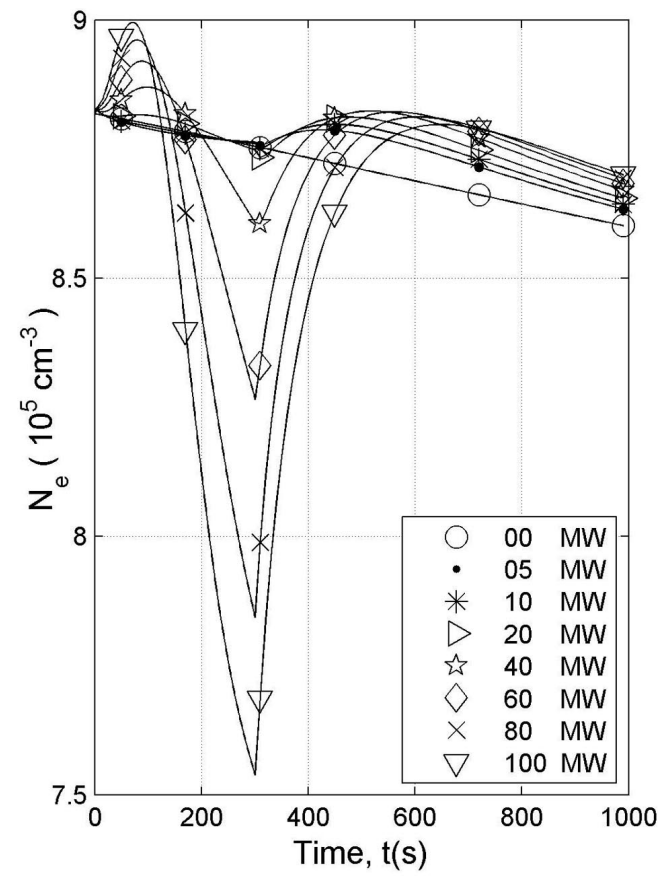

Fig. 8. The time variations of the electron concentration (in units of $10^{5} \mathrm{~cm}^{-3}$ ) at the level $h=235 \mathrm{~km}$. The results are given for the same cases as in Fig. 7.

\section{Summary and concluding remarks}

The mathematical model of the ionosphere, developed earlier, has been applied for simulations of the large-scale midlatitude F-layer modification by powerful HF radio waves, both for nocturnal and daytime conditions. The model produces the time variations of the electron density, positive ion velocity, and ion and electron temperature profiles within a magnetic field tube over an ionospheric heater. A part of the magnetic field tube of the ionospheric plasma is considered at distances between 100-700 km from the Earth along the magnetic field line. In the present study, our focus has been on the influence of the effective absorbed power (EAP) of heating HF waves on the large-scale F-region modification. Therefore, we have taken into account the main heating mechanism and neglected some specific effects of the heating process. Account has been taken only of the anomalous heating of the plasma in the presence of small-scale fieldaligned irregularities in the vicinity of the electron hybrid resonance frequency. We have taken advantage of the expression for the rate of anomalous heating, derived by Blaunshtein et al. (1992). This expression, in spite of its simplicity, permits to evaluate approximately the influence of the effective absorbed power on the large-scale F-region modification.

Despite of approximate description of heating mechanism caused by the action of the powerful HF radio waves, the applied mathematical model of the ionosphere is rather adequate. The model is based on numerical solution of the 
system of transport equations, which consists of the continuity equation, equation of motion for ion gas, and heat conduction equations for ion and electron gases. The equations provide the direct and resonantly scattered EUV (extreme ultraviolet) solar radiation, energy-dependent chemical reactions, frictional force between ions and neutrals, accelerational and viscous forces of ion gas, thermal conductions of electron and ion gases, heating due to ion-neutral friction, Joule heating, heating due to solar EUV photons, and electron energy losses due to elastic and inelastic collisions. The model takes into account the magnetization of the plasma at F-layer altitudes, drift of the ionospheric plasma, and geomagnetic field inclination, not always included in other models. Therefore, it may be expected that the model allows us to evaluate correctly the influence of the absorbed energy of heating HF waves on the large-scale F-region modification.

Our simulations were performed for the point with geographic coordinates of the "Sura" heating facility (Nizhny Novgorod, Russia) for autumn conditions. The heating facility was supposed to operate continuously during the period of five minutes. The frequency of powerful HF waves was chosen to be close to the most effective frequency for the largescale F2-layer modification. The calculations were made for distinct cases, in which the effective absorbed power has different values belonging to the 5-100 MW range.

The results of simulations indicated that, at levels where the wave frequency is close to the frequency of the electron hybrid resonance, a pronounced peak arises in the electron temperature profile due to the great energy input from the powerful HF wave. As a consequence, upward and downward ionospheric plasma fluxes arise from the level where the electron temperature peak is located. These fluxes produce visible changes in the electron concentration profile. The electron concentration can decrease in a wide height range including the level of the F2-layer peak.

It turned out that the value of the effective absorbed power can significantly influence the F-layer response to highpower radio waves in the mid-latitude ionosphere. The more the effective absorbed power (EAP) is, the higher the values of maximal amplitudes of variations of ionospheric quantities, produced by HF heating, ought to be. The calculated changes in the large-scale structure of the nocturnal F2 layer turned out to be more than those obtained under daytime conditions. When the EAP is $100 \mathrm{MW}$, powerful HF waves can lead to a decrease of more than $47 \%$ in electron concentration at the level of the F2-layer peak for nocturnal conditions while, in the daytime, this decrease may be of about $13 \%$, on condition that the duration of the heating facility action is five minutes.

Acknowledgements. This work was partly supported by the RFBR grant 10-01-00451. The authors would like to thank the referees for qualitative comments and helpful advice that led to improvement in the original manuscript.
Topical Editor M. Pinnock thanks three anonymous referees for their help in evaluating this paper.

\section{References}

Ashrafi, M., Kosch, M. J., Kaila, K., and Isham, B.: Spatiotemporal evolution of radio wave pump-induced ionospheric phenomena near the fourth electron gyroharmonic, J. Geophys. Res., 112, A05314, doi:10.1029/2006JA011938, 2007.

Belikovich, V. V., Benediktov, E. A., Tolmacheva, A. V., and Bakhmet'eva, N. V.: An investigation of the ionosphere with the help of artificial periodic inhomogeneities (in Russian), Institute of Applied Physics of the Russian Academy of Sciences, Nizhny Novgorod, Russia, 1999.

Blaunshtein, N. Sh., Vas'kov, V. V., and Dimant, Ya. S.: Resonance heating of the $\mathrm{F}$ region by a powerful radio wave, Geomagnetism and Aeronomia, 32, 95-99, 1992 (Russian issue).

Clausen, L. B. N., Yeoman, T. K., Wright, D. M., Robinson, T. R., Dhillon, R. S., and Gane, S. C.: First results of a ULF wave injected on open field lines by Space Plasma Exploration by Active Radar (SPEAR), J. Geophys. Res., 113, A01305, doi:10.1029/2007JA012617, 2008.

Dhillon, R. S., Robinson, T. R., and Yeoman, T. K.: EISCAT Svalbard radar observations of SPEAR-induced E- and F-region spectral enhancements in the polar cap ionosphere, Ann. Geophys., 25, 1801-1814, doi:10.5194/angeo-25-1801-2007, 2007.

Djuth, F. T., Thide, B., Ierkic, H. M., and Sulzer, M. P.: Large F-region electron-temperature enhancements generated by highpower HF radio waves, Geophys. Res. Lett., 14, 953-956, 1987.

Duncan, L. M., Sheerin, J. P., and Behnke, R. A.: Observations of ionospheric cavities generated by high-power radio waves, Phys. Rev. Lett., 61, 239-242, 1988.

Frolov, V. L., Eruchimov, L. M., Metelev, S. A., and Sergeev, E. N.: Temporal behavior of artificial small-scale irregularities: Review of experimental results, J. Atmos. Terr. Phys., 59, 2317-2324, 1997.

Gordon, W. E. and Carlson, H. C.: Arecibo heating experiments, Radio Sci., 9, 1041-1047, 1974.

Gustavsson, B., Sergienko, T., Rietveld, M. T., Honary, F., Steen, A., Brandstrom, B. U. E., Leyser, T. B., Aruliah, A. L., Aso, T., Ejiri, M., and Marple, S.: First tomographic estimate of volume distribution of HF-pump enhanced airglow emission, J. Geophys. Res., 106, 29105-29124, 2001.

Hansen, J. D., Morales, G. J., Duncan, L. M., and Dimonte, G.: Large-scale HF-induced ionospheric modification: experiments, J. Geophys. Res., 97, 113-122, 1992.

Heppner, J. P.: Empirical models of high-latitude electric field, J. Geophys. Res., 82, 1115-1125, 1977.

Honary, F., Stocker, A. J., Robinson, T. R., Jones, T. B., and Stubbe, $\mathrm{P}$.: Ionospheric plasma response to $\mathrm{HF}$ radio waves operating at frequencies close to the third harmonic of the electron gyrofrequency, J. Geophys. Res., 100, 21489-21501, 1995.

Jones, T. B., Robinson, T. R., Stubbe, P., and Kopka, H.: EISCAT observations of the heated ionosphere, J. Atmos. Terr. Phys., 48, 1027-1035, 1986.

Kosch, M. J., Pedersen, T., Rietveld, M. T., Gustavsson, B., Grach, S. M., and Hagfors, T.: Artificial optical emissions in the highlatitude thermosphere induced by powerful radio waves: An observational review, Adv. Space Res., 40, 365-376, 2007. 
Mantas, G. P.: Large 6300- $\AA$ airglow intensity enhancements observed in ionosphere heating experiments are exited by thermal electrons, J. Geophys. Res., 99, 8993-9002, 1994.

Mantas, G. P., Carlson, H. C., and La Hoz, C. H.: Thermal response of F-region ionosphere in artificial modification experiments by HF radio waves, J. Geophys. Res., 86, 561-574, 1981.

Meriwether, J. M., Heppner, J. P., Stolaric, J. D., and Wescott, E. M.: Neutral winds above $200 \mathrm{~km}$ at high latitudes, J. Geophys. Res., 78, 6643-6661, 1973.

Mingaleva, G. I. and Mingalev, V. S.: The formation of electron-temperature hot spots in the main ionospheric trough by the internal processes, Ann. Geophys., 14, 816-825, doi:10.1007/s00585-996-0816-x, 1996.

Mingaleva, G. I. and Mingalev, V. S.: Response of the convecting high-latitude F layer to a powerful HF wave, Ann. Geophys., 15, 1291-1300, doi:10.1007/s00585-997-1291-8, 1997.

Mingaleva, G. I., Mingalev, V. S., and Mingalev, I. V.: Simulation study of the high-latitude F-layer modification by powerful HF waves with different frequencies for autumn conditions, Ann. Geophys., 21, 1827-1838, doi:10.5194/angeo-211827-2003, 2003.

Mingaleva, G. I., Mingalev, V. S., and Mingalev, I. V.: Model prediction of the most effective frequency for the large-scale modification of the mid-latitude ionospheric $\mathrm{F}_{2}$ layer by powerful $\mathrm{HF}$ radio waves, Geomagn. Aeronomy, 48, 66-74, 2008.

Mingaleva, G. I., Mingalev, V. S., and Mingalev, I. V.: Model simulation of the large-scale high-latitude F-layer modification by powerful HF waves with different modulation, J. Atmos. Sol.Terr. Phy., 71, 559-568, 2009.
Pedersen, T., Esposito, R., Kendall, E., Sentman, D., Kosch, M., Mishin, E., and Marshall, R.: Observations of artificial and natural optical emissions at the HAARP facility, Ann. Geophys., 26, 1089-1099, doi:10.5194/angeo-26-1089-2008, 2008.

Richmond, A. D.: Electric field in the ionosphere and plasmasphere on quiet days, J. Geophys. Res., 81, 1447-1450, 1976.

Richmond, A. D., Blanc, M., Emery, B. A., Wand, R. H., Fejer, B. G., Woodman, R. F., Ganguly, S., Amayenc, P., Behnke, R. A., Calderon, C., and Evans, J. V.: An empirical model of quiet-day ionospheric electric fields at middle and low latitudes, J. Geophys. Res., 85, 4658-4664, 1980.

Rietveld, M. T., Kosch, M. J., Blagoveshchenskaya, N. F., Kornienko, V. A., Leyser, T. B., and Yeoman, T. K.: Ionospheric electron heating, optical emissions, and striations induced by powerful HF radio waves at high latitudes: Aspect angle dependence, J. Geophys. Res., 108, 1141, doi:10.1029/2002JA009543, 2003.

Robinson, T. R., Honary, F., Stocker, A. J., Jones, T. B., and Stubbe, P.: First EISCAT observations of the modification of F-region electron temperatures during RF heating at harmonics of the electron gyro frequency, J. Atmos. Terr. Phys., 58, 385-395, 1996.

Utlaut, W. F. and Violette, E. J.: A summary of vertical incidence radio observations of ionospheric modification, Radio Sci., 9, 895903, 1974.

Yeoman, T. K., Blagoveshchenskaya, N., Kornienko, V., Robinson, T. R., Dhillon, R. S., Wright, D. M., and Baddeley, L. J.: SPEAR: early results from a very high latitude ionospheric heating facility, Adv. Space Res., 40, 384-389, 2007. 